\title{
Positive outcome expectancy mediates the relationship between social influence and Internet addiction among senior high-school students
}

\author{
MIN-PEI LIN ${ }^{1} *$, JO YUNG-WEI WU ${ }^{2}$, CHAO-JUI CHEN ${ }^{1}$ and JIANING YOU ${ }^{3}$ \\ ${ }^{1}$ Department of Educational Psychology and Counseling, National Taiwan Normal University, Taipei City, Taiwan \\ ${ }^{2}$ Department of Counseling and Guidance, National University of Tainan, Tainan, Taiwan \\ ${ }^{3}$ Center for Studies of Psychological Application \& School of Psychology, South China Normal University, Guangzhou, China
}

(Received: March 1, 2018; revised manuscript received: May 16, 2018; accepted: May 25, 2018)

\begin{abstract}
Background and aims: Based on the foundations of Bandura's social cognitive theory and theory of triadic influence (TTI) theoretical framework, this study was designed to examine the mediating role of positive outcome expectancy of Internet use in the relationship between social influence and Internet addiction (IA) in a large representative sample of senior high-school students in Taiwan. Methods: Using a cross-sectional design, 1,922 participants were recruited from senior high schools throughout Taiwan using both stratified and cluster sampling, and a comprehensive survey was administered. Results: Structural equation modeling and bootstrap analyses results showed that IA severity was significantly and positively predicted by social influence, and fully mediated through positive outcome expectancy of Internet use. Discussion and conclusions: The results not only support Bandura's social cognitive theory and TTI framework, but can also serve as a reference to help educational agencies and mental health organizations design programs and create policies that will help in the prevention of IA among adolescents.
\end{abstract}

Keywords: Internet addiction, positive outcome expectancy of Internet use, social cognitive theory, social influence, theory of triadic influence

\section{INTRODUCTION}

Use of the Internet continues to rapidly spread, and has become a popular academic and leisure tool among adolescents around the world $(\mathrm{Wu}, \mathrm{Ko}$, Wong, $\mathrm{Wu}, \&$ Oei, 2016). Limited and appropriate Internet use is beneficial, but excessive and uncontrolled Internet use has frequently been linked to many maladaptive problems, in which the misuse and abuse of the Internet can lead to Internet addiction (IA; Liang, Zhou, Yuan, Shao, \& Bian, 2016). Young (2007) has identified IA as a new unrecognized clinical disorder that greatly affects a user's ability to control his/her online use and causes relationship, occupational, and social problems. In addition, some students have displayed symptoms of IA that include loss of control, preoccupation, craving, high tolerance for use of the Internet, withdrawal, a reduction in the ability to make decisions, and impairment of function (Ko, Yen, Chen, Chen, \& Yen, 2005). The reported ratios of IA varied widely (Cheng \& Li, 2014; Kuss, Griffiths, Karila, \& Billieux, 2014), but there is no doubt that IA has become an emerging social, education, and mental health issue around the world, especially among the adolescents (Zhao et al., 2017). Therefore, it is important for the educational agencies and mental health professionals to construct suitable IA prevention programs geared toward adolescents. It becomes more essential to understand the developing process of the Internet behavior, so that researchers can examine reciprocal links between outcomes and antecedents of the exposure (Eastin, 2005). There is an imperative need to identify the psychosocial risk factors and the process of IA.

Over the past few decades, we have seen mounting evidence in the usefulness of Bandura's social cognitive theory to explain addictive behaviors (Bandura, 1986; Lin, Ko, \& Wu, 2008); in particular, positive outcome expectancies are commonly defined as beliefs perceived by an individual on the likelihood of a behavior leading to some desired outcome (Bandura, 1997; Wu, Ko, Wong, et al., 2016). Numerous studies have found that positive outcome expectancy was significantly associated with the use of alcohol (Anthenien, Lembo, \& Neighbors, 2017), heroin (Lee, Bowen, Oei, \& Yen, 2012), betel quid (Chang, Ko, Wu, \& Cheng, 2007), marijuana (Tucker et al., 2014), cigarette (Hruska et al., 2014), and gambling (Flack \& Morris, 2016). Accordingly, when applied to explaining IA, Lin, Ko, and $\mathrm{Wu}(2011)$ found that positive outcome expectancy of Internet use, among psychosocial risk factors, was a strong predictor of IA in 3,616 college students. Similarly, Brand, Laier, and Young (2014) also found that Internet use expectancies positively predicted IA among 1,019 online users. Subsequently, Lee, Ko, and Chou (2015)

\footnotetext{
* Corresponding author: Min-Pei Lin; Department of Educational Psychology and Counseling, National Taiwan Normal University, No. 162, Sec. 1, Heping E. Rd., Da-an District, Taipei City 106, Taiwan; Phone: +886 27734 3770; Fax: +886 22341 3865; E-mail: 1mmpp@ntnu.edu.tw
}

This is an open-access article distributed under the terms of the Creative Commons Attribution-NonCommercial 4.0 International License, which permits unrestricted use, distribution, and reproduction in any medium for non-commercial purposes, provided the original author and source are credited, a link to the CC License is provided, and changes - if any - are indicated. 
also discovered that Internet expectancy positively predicted IA in a large-scale national survey conducted by the Taiwan Ministry of Education among 25,573 junior and senior highschool students. A further study conducted by Wu incorporated a 1-year follow-up and revealed that the Internet use expectancy for tension reduction and disinhibition in time 1 was positively correlated with IA in time 2 among 623 college students (Wu, Ko, Tung, \& Li, 2016).

With the heightening risks associated with adolescents and addictive behaviors, the role of social influence also plays an important aspect in the development of addictive behaviors, including alcohol use (Simons-Morton et al., 2016), cigarette use (Dahlui et al., 2015), heroin and cocaine use (Bohnert, Bradshaw, \& Latkin, 2009), polysubstance use (Russell, Trudeau, \& Leland, 2015), gambling (Neighbors et al., 2015), and Internet gaming addiction (Wu, Ko, Wong, et al., 2016). Comprehensively, we incorporated the theory of triadic influence (TTI; Flay \& Petraitis, 1994; Petraitis, Flay, \& Miller, 1995) as a theoretical framework in attempt to examine the impact and predictability of social influence and positive outcome expectancy to IA. TTI integrates variables and processes from many psychological and sociological theories of behavior onset and change, and it provides a unified theoretical framework that incorporates influential factors or the causes of the behavior (Flay, Petraitis, \& $\mathrm{Hu}$, 1999). More recently, the TTI has been used as a framework in research to examine addictive behaviors of adolescents (Chun, 2015; Defoe, Semon Dubas, Somerville, Lugtig, \& van Aken, 2016; Grigsby, Forster, Unger, \& Sussman, 2016). The TTI organizes the influences on addictive behaviors into three substantive domains of influence (interpersonal stream, cultural/attitudinal stream, and intrapersonal stream) and discusses the extent to which different factors influence behavior as ranging from very proximal to distal and ultimate (Grigsby et al., 2016). In the theoretical flow hypothesized by the TTI, ultimate causes influence distal constructs, and distal constructs influence proximal predictors; this occurs not only within the same stream of influence, but also across the streams (Bavarian et al., 2014).

In the theoretical frameworks of TTI, social influence falls in the category of distal and ultimate determinants, while positive outcome expectancy is considered a proximal determinant (Chun, 2015; Klein Velderman et al., 2015). This study adopted the theoretical concepts of TTI, and hypothesized that positive outcome expectancy will mediate the relationship between social influence and IA. Therefore, the aim of this study was to examine the mediating role of positive outcome expectancy of Internet use in the relationship between social influence and IA in a large representative sample of senior high-school students. These findings can be used to help educational agencies and mental health organizations design programs and create policies that will help in the prevention of IA among adolescents.

\section{METHODS}

\section{Participants and procedure}

Participants were recruited from senior high schools throughout Taiwan using both stratified and cluster sampling. The sample was stratified by school type (i.e., regular high school or vocational high school) and clustered by class. Based on the 2012 school year data provided by Department of Statistics, Ministry of Education (https://stats.moe.gov.tw/qframe.aspx?qno=MQA1AA2), there were a total of 402,689 regular high-school students and 369,436 vocational high-school students in Taiwan (ratio of 52:48). According to the ratio, this study invited two regular high schools and two vocational high schools from October 2014 to December 2014, and selected 2,171 students (1,128 regular high-school students, yielding $51.95 \%$ of the total sample size that was close to the ratio of the population group). Of them, 1,922 students $(1,019$ females) participated, resulting in a response rate of $88.53 \%$. Since 31 participants did not complete the questionnaire, the final sample consisted of 1,891 students $\left(M_{\mathrm{age}}=16.83, S D=0.37 ; 53.36 \%\right.$ females $)$. Of the final sample, 924 participants were regular high-school students, and 967 participants were vocational high-school students. Administrators from the participating senior high schools received a copy of the survey and guidelines for administering the survey. Students were also fully informed of the purpose of the study and voluntary participation was emphasized. The questionnaires were administered in classrooms.

\section{Measures}

Social Influence for Internet Use Scale. This scale was revised from the Parental Influence for Internet Gaming Addiction Scale and Peer Influence for Internet Gaming Addiction Scale (Wu, Ko, Wong, et al., 2016). In the theoretical frameworks of TTI, social influence, as distal and ultimate determinants, includes the contents of "substancespecific behaviors of influential role models (such as knowing and observing the smoking and/or drinking behavior of parents);" "general values that affect the attitudes of adolescents toward substance use;" and "an adolescent's immediate surroundings, such as neighbors, schools, and culture, including weak public policies on substance use." In this study, the "Social Influence for Internet Use Scale" contains three subscales, including "Perceived Frequency of Others' Internet Use," "Frequency of Others' Invitation to Use Internet," and "Internet Use Accessibility." Fourteen items were constructed to assess the three constructs, which encompass (a) perceived parental, surrounding relatives, siblings, peers, and friends Internet use frequencies (five items); (b) the frequency of parental, surrounding relatives, siblings, peers, and friends invitations to use Internet (five items); and (c) Internet use accessibility (four items, including "Can you use the Internet at home?," "Can you use the Internet at school?," "Is there an Internet Café near your home or school?," and "Do you often see commercials or media that encourage you to use the Internet?"). Higher scores indicated a higher perceived frequency of others' Internet use, higher frequencies of others invitations to use the Internet, and easier Internet use accessibility. In this study, the Cronbach's $\alpha$ value of this scale and the three subscales were $.77, .74, .79$, and .52 , respectively.

Positive Outcome Expectancy of Internet Use Questionnaire (POEIUQ). This scale consisted of 26 items 
and was measured using a 6-point Likert scale (Lin, Wu, You, Hu, \& Yen, 2018). A higher total score represented a greater degree in positive outcome expectancy of Internet use among senior high-school students. The internal consistency coefficient was .94 (Lin et al., 2018). The questionnaire consists of four subscales: "Self-Achievement" (eight items), "Acquire Information" (six items), "Stress Reduction and Pleasurable" (six items), and "Interpersonal Connection" (six items). In addition, confirmatory factor analysis supported evidence of factorial validity (fourfactor solution fitted the data most effectively) for the scales (Lin, $\mathrm{Hu}, \mathrm{Lin}, \& \mathrm{Wu}, 2013$ ). In this study, the Cronbach's $\alpha$ value of the four subscales were $.92, .95$, .93 , and .93 , respectively.

Chen's Internet Addiction Scale (CIAS). The CIAS is a 26-item self-reported questionnaire measured using a 4point Likert scale (Chen, Weng, Su, Wu, \& Yang, 2003), and a higher total score means greater levels of IA. The CIAS contained five subscales, including "compulsive use of Internet" (five items), "withdrawal symptoms of IA" (five items), "tolerance symptoms of IA" (four items), "interpersonal and health-related problems of IA" (seven items), and "time management problems" (five items). The internal consistency coefficients of each subscale ranged from .78 to .81 , and the split-half reliability over 2 weeks is 0.83 (Chen et al., 2003). Moreover, correlation analyses yielded a significant positive correlation of the total scale and subscale scores of the CIAS to the hours spent weekly on Internet activity (Chen et al., 2003), indicating that CIAS has a good criterion-related validity. In addition, this scale has also been successfully used among senior high-school students in Taiwan (Chang, Chiu, Lee, Chen, \& Miao, 2014).

\section{Statistical analyses}

The SPSS for Windows version 18.0 was used for data analyses, and the significant level was set at $p<.05$. Initial descriptive statistics were compiled, and Pearson's correlation was used to identify the relationship among all measures. Furthermore, structural equation modeling (SEM) analyses with the robust maximum likelihood method were carried out by AMOS 18.0 to examine the mediating effect of positive outcome expectancy of Internet use between social influence and IA severity. In addition, we also used Sobel test and bootstrap analyses to test the mediation model, respectively (Mackinnon, Lockwood, Hoffman, West, \& Sheets, 2002; Shrout \& Bolger, 2002).

\section{Ethics}

The study procedures were carried out in accordance with the Declaration of Helsinki. The study protocol was approved by the review board of the Ministry of Science and Technology in Taiwan. Before conducting the study, we obtained full consent from the principals, and the counseling and guidance team of each senior high school. In addition, teachers of the participating classes gave consent prior to administrating the survey battery. All the participants were informed about the study and all provided informed consent.

\section{RESULTS}

\section{Pearson's correlations among variables}

The correlations presented in Table 1 showed that total social influence and its three subscales were significantly and positively correlated with IA severity and total POEIUQ and its four subscales, respectively. Moreover, total POEIUQ and its three subscales and IA severity were positively correlated with one another, respectively. However, no significant correlation was uncovered between the Acquire Information subscale of POEIUQ and IA severity. Thus, the Acquire Information subscale of POEIUQ was removed in the SEM analysis.

\section{The mediating role of positive outcome expectancy of Internet use}

Using the SEM by the robust maximum likelihood method, the hypothesized models presented in Figure 1 were examined. The model yielded a significant $\chi^{2}$ value: $\chi^{2}(41, N=1,891)=575.83, p<.001$. However, given that a non-significant $\chi^{2}$ is hard to achieve with large sample sizes, the fit of the models was interpreted on the basis of a range of other fit indices. The AMOS program produces a range of goodness of fit indices including outputs for the comparative fit index (CFI), incremental fit index (IFI), the Bentler-Bonett normed fit index (NFI), and Bentler-Bonett non-normed fit index (NNFI), which consider the degrees of freedom of the model. If these values are greater than 0.90 , they are regarded to be acceptable by convention ( $\mathrm{Hu} \&$ Bentler, 1999). The root mean square error of approximation (RMSEA) is based on the population error of approximation measures discrepancy per degree of freedom; a value less than 0.08 would indicate a reasonable error of approximation (Browne \& Cudeck, 1993). The other fit indices in the models were 0.94 on the CFI, 0.94 on the IFI, 0.93 on the NFI, 0.92 on the NNFI, and 0.08 on the RMSEA $(90 \%$ confidence interval is between 0.077 and 0.089 ). The measurement model demonstrated a good fit. Therefore, the overall fit of the mediational model was adequate and able to explain $13.4 \%$ of the variance.

The mediational model presented in Figure 1 exhibited that social influence had a significant effect on IA severity and positive outcome expectancy of Internet use, respectively. Moreover, the effect of social influence to IA severity decreased from $0.29(p<.001)$ to $0.06(p>.05)$ when the analysis included the positive outcome expectancy of Internet use effects. In addition, the Sobel test (MacKinnon et al., 2002) indicated that the mediated effect was significant for "social influence - positive outcome expectancy of Internet use - IA severity" $(z=6.78, p<.001)$, demonstrating a full mediating effect from social influence to IA severity through positive outcome expectancy of Internet use.

Furthermore, we also used the bootstrap analyses to examine the indirect effect of social influence on IA severity via positive outcome expectancy of Internet use (Shrout \& Bolger, 2002). As recommended by Mallinckrodt, Abraham, Wei, and Russell (2006), 10,000 bootstrap samples from the data set were created, and a bias-corrected $95 \%$ confidence interval for the estimates of the direct and indirect effects 


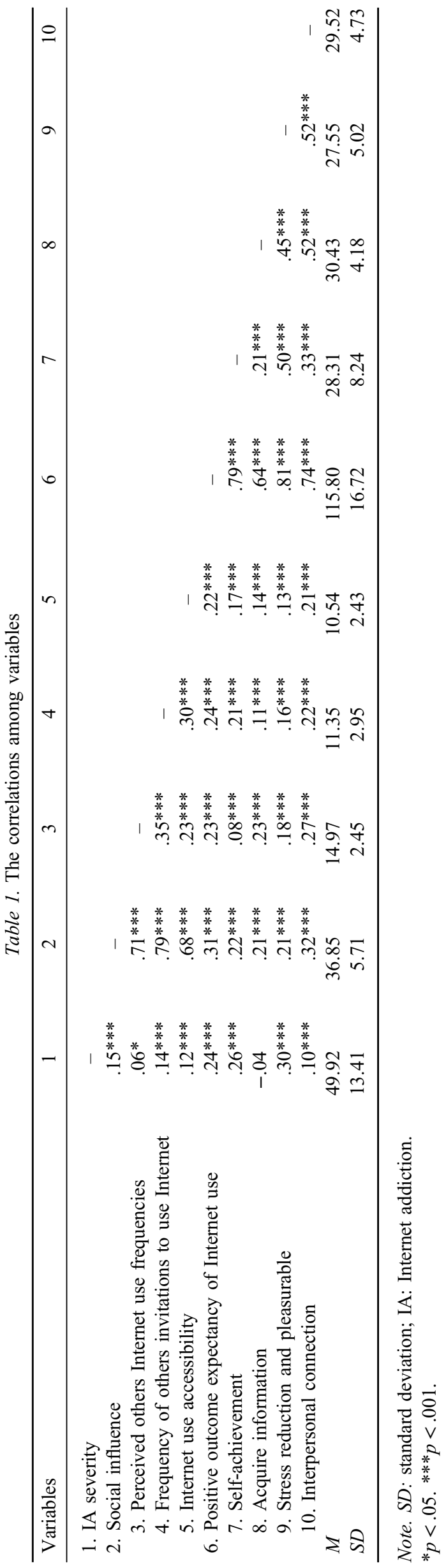

was used. The results indicated that the mean indirect (unstandardized) effect of social influence on IA severity via positive outcome expectancy of Internet use was 0.27 , and bias-corrected $95 \%$ confidence interval was between 0.20 and 0.37 , which did not include zero, thus the indirect effect was statistically significant $(p<.001)$. However, the mean direct (unstandardized) effect of social influence on IA severity was 0.14 , and biascorrected $95 \%$ confidence interval was between -0.04 and 0.26 , which included zero and therefore the direct effect was statistically non-significant. The full mediation model was confirmed.

\section{DISCUSSION}

Consistent with previous studies, this study found that social influence was correlated with IA severity (Wu, Ko, Wong, et al., 2016), and positive expectancy of Internet use was also correlated with IA severity (Brand et al., 2014; Lee et al., 2015; Lin et al., 2011; Wu, Ko, Tung, et al., 2016). Furthermore, this study found that social influence was able to predict the increase of IA severity; and social influence also predicted an increase of positive outcome expectancy of Internet use, which played a full mediation role between social influence and IA in the sampled senior high-school students.

Regarding the association between positive outcome expectancy of Internet use and IA among senior high-school students, bivariate correlations showed that the total POEIUQ and its three subscales (i.e., Self-Achievement, Stress Reduction and Pleasurable, and Interpersonal Connection) were significantly associated with IA, but the Acquire Information subscale was not. To our knowledge, this was the first study to point out that not all of the constructs in the positive outcome expectancy of Internet use are significantly associated with IA. This result is similar to that of Young (1998), which indicated that a majority of Internet addicts use the Internet for online gaming and communication, and for the purpose of gaining pleasure and happiness. On the other hand, non-Internet addicts use the Internet mainly for information seeking and to send/ receive e-mails for school or work-related communication. Thus, when Internet users have positive expectations of Acquired Information, and believe that "Going online allows me to easily gain more new information or can acquire knowledge from different areas," they are less likely to be vulnerable to the risk of developing IA, and may perceive the Internet as an instrument rather than a platform for managing their positive/negative emotions. However, if users use the Internet for the purpose of "Self-Achievement" or "Stress Reduction and Pleasurable," in which the user goes online in order to enhance his sense of accomplishment or gain pleasure, then, there is a higher chance of developing IA. This finding is in accordance with $\mathrm{Wu}, \mathrm{Ko}$, Tung, et al.'s (2016) study on college students, which discovered that students who often use the Internet for tension reduction (such as temporarily forgetting their problems or to feel pleasure) result in a higher risk of developing IA. Future research should examine the constructs of positive outcome expectancy of Internet use among different age groups and 


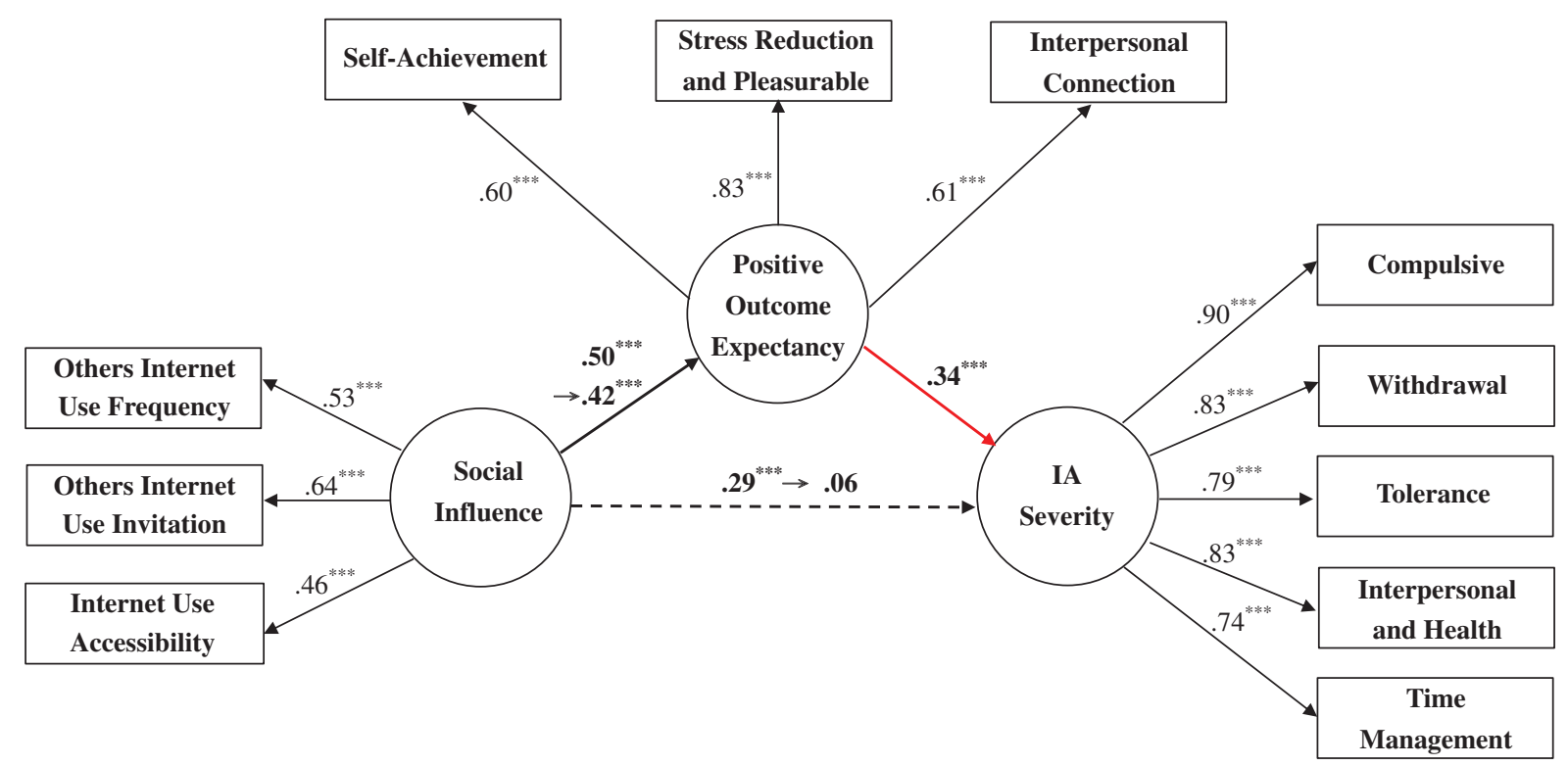

Figure 1. Mediational model. $\chi^{2}: 575.83 * * *(d f: 41)$; CFI: 0.94; IFI: 0.94; NFI: 0.93; NNFI: 0.92; RMSEA: $0.08 ;{ }^{* * *} p<.001$

its relationship to IA. For instance, can the positive outcome expectancy of academic or ubiquitous learning through the Internet be a protective factor for IA?

After eliminating the Acquire Information subscale, this study found that positive outcome expectancy of Internet use positively predicted IA. Moreover, our results also showed that the relationship between social influence and IA was fully mediated by the positive outcome expectancy of Internet use. That is to say, social influence is associated with higher levels of positive outcome expectancy of Internet use, which is then associated with an increase of IA severity. This finding is similar to the results of previous literatures (Wood, Read, Palfai, \& Stevenson, 2001; Wu, Ko, Wong, et al., 2016). In addition, this result not only supports the theoretical constructs of TTI (Flay \& Petraitis, 1994), but also is in accordance to Bandura's (1986) social cognitive theory. Bandura (1969) proposed the concept of vicarious learning (or social learning), which occurs by observing, retaining, and replicating the behaviors of others. Connors, O'Farrell, Cutter, and Thompson (1986) defined positive outcome expectancy and pointed out that one gains a positive experience from directly or indirectly coming in contact with someone in the process of an addictive behavior (such as observing or listening to a brother who says that smoking is a relaxing behavior). Such an experience transforms into an internal cognitive representation and forms a positive outcome expectancy of a certain behavior. Furthermore, based on Bandura's (1969) perspective, Masisito, Carey, and Bradizza (1999) believed that cognition served a mediating role in the relationship between the external environment (or event) and the individual. In other words, the external environment (or event) provides information that allows the individual to process their cognition and to proceed with the behavior; outcome expectancy serves an important role in the facilitating of future behaviors. Thus, high-school students who are situated in an environment that comes in constant contact with surrounding individuals who frequently use or invite one to use the Internet or provides easy access to the Internet are more likely to develop positive cognitive expectancies about Internet use, and increase the chance of becoming Internet-addicted.

Eastin (2005) conducted research on Internet use and stated that peers who have successful experiences in information search and entertainment on the Internet (such as listening to music and watching movies online) will gain more positive outcome expectancies in these aspects. In addition, an individual will gain a more positive experience about the Internet when they observe a higher ratio of Internet use among their surrounding individuals, and in turn enhance positive outcome expectancy and increases Internet use. In other words, through the observation of positive Internet use experiences of surrounding individuals, high-school students can be imperceptibly influenced to develop a positive outcome expectancy of Internet use and thus increases their Internet-use behavior and risk of IA.

The results from this study provide evidence in explaining how the positive outcome expectancy of Internet use fully mediated the relationship between social influence and IA severity. In order to minimize the rate of IA among senior high-school campuses, preventive programs should focus on educating students on the positive and negative outcomes of IA in order to decrease the positive expectancies of Internet use (Wu, Ko, Tung, et al., 2016). In an attempt to prevent IA among adolescents, educational agencies and mental health organizations should emphasize plans to try to reduce positive outcome expectancy with regard to Internet use using methods, such as multisession experiential expectancy challenges (Lin et al., 2018). In addition, apart from using the Internet for "Stress reduction and Pleasurable" or for "Self-Achievement," concrete strategies need to be taught to allow students to experience other non-online activities that can effectively help them in stress reduction and pleasure or able self-achievement, reducing their chance of being at risk of IA.

In relevance to the theoretical framework of TTI, ultimate causes influence distal constructs, and distal constructs influence proximal predictors. Social influence corresponds to the constructs of TTI, which include substance-specific 
behaviors of influential role models (such as "knowing and observing the smoking and/or drinking behavior of parents"); "general values that affect the attitudes of adolescents toward substance use," and "an adolescent's immediate surroundings, such as neighbors, schools, and culture, including weak public policies on substance use," all are aspects of distal and ultimate determinants. Positive outcome expectancy can be classified as proximal determinants (Chun, 2015; Klein Velderman et al., 2015). This study found that the positive outcome expectancy of Internet use was able to fully mediate the predictive relationship between social influence and IA, which fits the theoretical framework of TTI. In the social culture of Taiwan, the education system and mass media have created a social atmosphere that the "Internet is a basic necessity," and thus facilitates people in Taiwan to have a high acceptance of Internet behavior. Individuals constantly having a smartphone or tablet beside them can be seen frequently and perceived as a normal behavior by society. Thus, the social values brought upon the Internet behaviors have greatly influenced the cognition and expectancies of the adolescent population. When the majority of the society goes online, there is a perceived social acceptance of the Internet behavior, and enhances the positive outcome expectancy in increasing Internet use and heightening the risk of IA.

From an ecological perspective of TTI, IA is not only a personal problem, but also an important social phenomenon that needs to be improved. We need to broaden our angle of vision from the individual perspective to a higher end, and address the process of IA through each of its layers, from the external influences and values of the social environment (distal and ultimate determinants), to the internal influences in positive outcome expectancy of Internet use (proximal determinants), in its development of IA risk among adolescents.

On the other hand, previous research has pointed out that the multifaceted nature and heterogeneity of behavioral addictions are too often neglected in favor of a simplistic symptomatic description (Billieux, Schimmenti, Khazaal, Maurage, \& Heeren, 2015). Starcevic (2010) indicated that IA appears to be too heterogeneous as a concept, which consists of at least three subtypes of excessive gaming, problematic sexual behavior, and text messaging. Different purposes of using the Internet may be associated with different patterns of use, different underlying psychopathology, and different adverse consequences. Furthermore, Starcevic (2013) pointed out that IA is a misleading term and should be replaced by addictions to Internet-related activities, if the pattern of such activities meets the criteria of behavioral addiction. Recent trends in psychiatric nosology have generally favored narrower and more homogenous terms such as online gaming addiction (i.e., Internet gaming disorder instead of Internet use disorder in the DSM-5) over a broader, heterogeneous term such as IA (Starcevic \& Billieux, 2017). Hence, it is reasonable to expect that future research will see a move away from IA in general toward addictions to specific online behaviors (Starcevic \& Aboujaoude, 2017). We suggest that future studies focus on different online behavior addictions in order to understand the specific positive outcome expectancy in the relationship and to verify Bandura's social cognitive theory and TTI theoretical framework in the application of different online behavior addictions.

This study highlighted the association between social influence and IA and undertook to provide a possible underlying process in the relationship. However, several limitations should be considered. First, regarding the mediating model, this study is a cross-sectional design in nature; thus, the causal relationship between social influence, positive outcome expectancy of Internet use, and IA cannot be fully illuminated. We suggest future research to conduct follow-up studies to further understand the influential process in the relationship. Second, in spite of a large sample size, all the participants consisted of senior high-school students in Taiwan, and only a total of four schools were selected as sampling points. Therefore, generalizations of our findings to different school types, and other age groups and countries or cultures may need further consideration. Third, the constructs of positive outcome expectancy of Internet use may be different among the age groups as well as different regions in the world, and the different constructs can also have varying correlations with IA. Thus, future research can recruit different age groups among differing regions in the world in order to construct a more universal POEIUQ to understand and compare the relationship between the content constructs and IA. Finally, the full mediation model was able to explain $13.4 \%$ variance of IA severity in the total sample, indicating that additional factors may also play part in the learning process, such as the TTI theoretical framework and other variables (such as selfesteem, emotional instability, and refusal self-efficacy, etc.), which can influence the relationship between social influence and IA severity, and should be incorporated to replicate the mediation model.

\section{CONCLUSIONS}

Based on the foundation of Bandura's social cognitive theory and TTI theoretical framework, this study was designed to examine social influence and the positive outcome expectancy of Internet use and their contribution to IA among senior high-school students using SEM and bootstrap analyses in a cross-sectional study design. The goal of this study was to determine whether the relationship between social influence and IA is mediated by positive outcome expectancy of Internet use. The results indicated that social influence was able to positively predict IA severity in a large representative sample of senior high-school students. This relationship, however, was fully mediated by the positive outcome expectancy of Internet use. The results are in accordance with Bandura's social cognitive theory in which cognitive factor is a mediating variable between the relationship of the external environment or event and the individual. In addition, the mechanism behind vicarious learning allows us to explain how highschool students are effected by social influence and in turn influences their positive attitude toward Internet use and increases the risk of IA. Furthermore, the results from this study were also in accordance with the theoretical framework of TTI. Following the theoretical perspective that ultimate causes influence distal constructs, and distal 
constructs influence proximal predictors, it is probable that the high usage rate and acceptance of the Internet by the social culture greatly enhanced the positive outcome expectancy of Internet use among high-school students, and in turn increases their Internet use and risk of developing IA.

Funding sources: This study was supported in part by the Ministry of Science and Technology in Taiwan (grant no.: MOST 106-2511-S-003-034-MY2 and MOST 102-2511-S003-016-MY3).

Authors' contribution: M-PL: study concept and design, analysis and interpretation of data, statistical analysis, obtained funding, development of the manuscript, revision and overall approval of the final manuscript, and study supervision. JY-WW: study concept and design, analysis and interpretation of data, development of the manuscript, and revision. C-JC: study concept and design, analysis and interpretation of data, statistical analysis, and data gathering. JY: study concept and design, analysis and interpretation of data, and statistical analysis. All authors had full access to all data in the study and take responsibility for the integrity of the data and the accuracy of the data analysis.

Conflict of interest: The authors declare no conflict of interest.

\section{REFERENCES}

Anthenien, A. M., Lembo, J., \& Neighbors, C. (2017). Drinking motives and alcohol outcome expectancies as mediators of the association between negative urgency and alcohol consumption. Addictive Behaviors, 66, 101-107. doi:10.1016/j. addbeh.2016.11.009

Bandura, A. (1969). Principles of behavior modification. New York, NY: Holt, Rinehart \& Winston.

Bandura, A. (1986). Social foundations of thought and action: A social cognitive theory. Englewood Cliffs, NJ: Prentice Hall.

Bandura, A. (1997). Self-efficacy: The exercise of control. New York, NY: W. H. Freeman.

Bavarian, N., Flay, B. R., Ketcham, P. L., Smit, E., Kodama, C., Martin, M., \& Saltz, R. F. (2014). Using structural equation modeling to understand prescription stimulant misuse: A test of the theory of triadic influence. Drug and Alcohol Dependence, 138, 193-201. doi:10.1016/j.drugalcdep.2014.02.700

Billieux, J., Schimmenti, A., Khazaal, Y., Maurage, P., \& Heeren, A. (2015). Are we overpathologizing everyday life? A tenable blueprint for behavioral addiction research. Journal of Behavioral Addictions, 4(3), 119-123. doi:10.1556/2006.4.2015.009

Bohnert, A. S., Bradshaw, C. P., \& Latkin, C. A. (2009). A social network perspective on heroin and cocaine use among adults: Evidence of bidirectional influences. Addiction, 104(7), 1210-1218. doi:10.1111/j.1360-0443.2009.02615.x

Brand, M., Laier, C., \& Young, K. S. (2014). Internet addiction: Coping styles, expectancies, and treatment implications. Frontiers in Psychology, 5, 1256. doi:10.3389/fpsyg.2014.01256

Browne, M. W., \& Cudeck, R. (1993). Alternative ways of assessing model fit. In K. A. Bollen \& J. S. Long (Eds.),
Testing structural equation models (pp. 136-162). Newbury Park, CA: Sage.

Chang, C. H., Ko, H. C., Wu, J. Y. W., \& Cheng, C. P. (2007). Social cognitive determinants of betel quid chewing among college students in southern Taiwan: A revised attitudesocial influence-efficacy model. Addictive Behaviors, 32(10), 2345-2350. doi:10.1016/j.addbeh.2007.02.001

Chang, F. C., Chiu, C. H., Lee, C. M., Chen, P. H., \& Miao, N. F. (2014). Predictors of the initiation and persistence of Internet addiction among adolescents in Taiwan. Addictive Behaviors, 39(10), 1434-1440. doi:10.1016/j.addbeh.2014.05.010

Chen, S. H., Weng, L. C., Su, Y. J., Wu, H. M., \& Yang, P. F. (2003). Development of Chinese Internet Addiction Scale and its psychometric study. Chinese Journal of Psychology, 45, 279-294. doi:10.1037/t44491-000

Cheng, C., \& Li, A. Y. (2014). Internet addiction prevalence and quality of (real) life: A meta-analysis of 31 nations across seven world regions. Cyberpsychology, Behavior and Social Networking, 17(12), 755-760. doi:10.1089/cyber.2014.0317

Chun, J. S. (2015). Determinants of tobacco use among Korean female adolescents: Longitudinal test of the theory of triadic influence. Children and Youth Services Review, 50, 83-87. doi:10.1016/j.childyouth.2015.01.009

Connors, G. J., O'Farrell, T. J., Cutter, H. S., \& Thompson, D. L. (1986). Alcohol expectancies among male alcoholics, problem drinkers, and nonproblem drinkers. Alcoholism, Clinical and Experimental Research, 10(6), 667-671. doi:10.1111/j.15300277.1986.tb05165.x

Dahlui, M., Jahan, N. K., Majid, H. A., Jalaludin, M. Y., Murray, L., Cantwell, M., Su, T. T., \& Al-Sadat, N. (2015). Risk and protective factors for cigarette use in young adolescents in a school setting: What could be done better? PLoS One, 10(6), e0129628. doi:10.1371/journal.pone.0129628

Defoe, I. N., Semon Dubas, J., Somerville, L. H., Lugtig, P., \& van Aken, M. A. (2016). The unique roles of intrapersonal and social factors in adolescent smoking development. Developmental Psychology, 52(12), 2044-2056. doi:10.1037/dev0000198

Eastin, M. S. (2005). Teen Internet use: Relating social perceptions and cognitive models to behavior. Cyberpsychology, Behavior and Social Networking, 8(1), 62-75. doi:10.1089/cpb.2005.8.62

Flack, M., \& Morris, M. (2016). The temporal stability and predictive ability of the Gambling Outcome Expectancies Scale (GOES): A prospective study. Journal of Gambling Studies, 32(3), 923-933. doi:10.1007/s10899-015-9581-7

Flay, B. R., \& Petraitis, J. (1994). The influence of triadic influence: A new theory of health behavior with implications for preventive intervention. In G. Albrecht (Ed.), Advances in medical sociology: A reconsideration of health change models (Vol. 4, pp. 19-44). Greenwich, CT: JAI Press.

Flay, B. R., Petraitis, J., \& Hu, F.B. (1999). Psychosocial risk and protective factors for adolescent tobacco use. Nicotine \& Tobacco Research, 1, S59-S65. doi:10.1080/14622299050011611

Grigsby, T. J., Forster, M., Unger, J. B., \& Sussman, S. (2016). Predictors of alcohol-related negative consequences in adolescents: A systematic review of the literature and implications for future research. Journal of Adolescence, 48, 18-35. doi:10.1016/ j.adolescence.2016.01.006

Hruska, B., Bernier, J., Kenner, F., Kenne, D. R., Boros, A. P., Richardson, C. J., \& Delahanty, D. L. (2014). Examining the relationships between posttraumatic stress disorder symptoms, positive smoking outcome expectancies, and cigarette smoking 
in people with substance use disorders: A multiple mediator model. Addictive Behaviors, 39(1), 273-281. doi:10.1016/j. addbeh.2013.10.002

Hu, L., \& Bentler, P. (1999). Cutoff criteria for fit indices in covariance structure analysis: Conventional criteria versus new alternatives. Structural Equation Modeling, 6(1), 1-55. doi:10.1080/10705519909540118

Klein Velderman, M., Dusseldorp, E., van Nieuwenhuijzen, M., Junger, M., Paulussen, T. G., \& Reijneveld, S. A. (2015). Cultural, social and intrapersonal factors associated with clusters of co-occurring health-related behaviours among adolescents. European Journal of Public Health, 25(1), 31-37. doi:10.1093/eurpub/cku051

Ko, C. H., Yen, J. Y., Chen, C. C., Chen, S. H., \& Yen, C. F. (2005). Proposed diagnostic criteria of Internet addiction for adolescents. Journal of Nervous and Mental Disease, 193(11), 728-733. doi:10.1097/01.nmd.0000185891.13719.54

Kuss, D. J., Griffiths, M. D., Karila, L., \& Billieux, J. (2014). Internet addiction: A systematic review of epidemiological research for the last decade. Current Pharmaceutical Design, 20, 4026-4052. doi:10.2174/138161281131999 90617

Lee, K. H., Bowen, S., Oei, T. P., \& Yen, C. F. (2012). An expanded self-medication hypothesis based on cognitivebehavioral determinants for heroin abusers in Taiwan: A cross-sectional study. American Journal on Addictions, 21(6), 516-523. doi:10.1111/j.1521-0391.2012.00285.x

Lee, Y. H., Ko, C. H., \& Chou, C. (2015). Re-visiting Internet addiction among Taiwanese students: A cross-sectional comparison of students' expectations, online gaming, and online social interaction. Journal of Abnormal Child Psychology, 43(3), 589-599. doi:10.1007/s10802-014-9915-4

Liang, L., Zhou, D., Yuan, C., Shao, A., \& Bian, Y. (2016). Gender differences in the relationship between Internet addiction and depression: A cross-lagged study in Chinese adolescents. Computers in Human Behavior, 63, 463-470. doi:10.1016/j. chb.2016.04.043

Lin, M. P., Hu, W. H., Lin, Y. P., \& Wu, Y. W. (2013). Internet use related cognitions scale: Development, confirmatory factor validation and psychometric properties. Paper presented at the 44th Society for Psychotherapy Research International Annual Meeting, Brisbane, Australia.

Lin, M. P., Ko, H. C., \& Wu, Y. W. (2008). The role of positive/ negative outcome expectancy and refusal self-efficacy of Internet use on Internet addiction among college students in Taiwan. Cyberpsychology, Behavior and Social Networking, 11(4), 451-457. doi:10.1089/cpb.2007.0121

Lin, M. P., Ko, H. C., \& Wu, Y. W. (2011). Prevalence and psychosocial risk factors associated with Internet addiction in a nationally representative sample of college students in Taiwan. Cyberpsychology, Behavior and Social Networking, 14(12), 741-746. doi:10.1089/cyber.2010.0574

Lin, M. P., Wu, J. Y., You, J., Hu, W. H., \& Yen, C. F. (2018). Prevalence of Internet addiction and its risk and protective factors in a representative sample of senior high school students in Taiwan. Journal of Adolescence, 62, 38-46. doi:10.1016/j.adolescence.2017.11.004

Mackinnon, D. P., Lockwood, C. M., Hoffman, J. M., West, S. G., \& Sheets, V. (2002). A comparison of methods to test mediation and other intervening variable effect. Psychological Methods, 7, 83-104. doi:10.1037//1082-989X.7.1.83
Mallinckrodt, B., Abraham, W. T., Wei, M., \& Russell, D. W. (2006). Advances in testing the statistical significance of mediation effects. Journal of Counselling Psychology, 53(3), 372-378. doi:10.1037/0022-0167.53.3.372

Masisito, S. A., Carey, K. B., \& Bradizza, C. M. (1999). Social learning theory. New York, NY: Guilford Press.

Neighbors, C., Rodriguez, L. M., Rinker, D. V., Gonzales, R. G., Agana, M., Tackett, J. L., \& Foster, D. W. (2015). Efficacy of personalized normative feedback as a brief intervention for college student gambling: A randomized controlled trial. Journal of Consulting and Clinical Psychology, 83(3), 500-511. doi:10.1037/a0039125

Petraitis, J., Flay, B. R., \& Miller, T. Q. (1995). Reviewing theories of adolescent substance use: Organizing pieces in the puzzle. Psychological Bulletin, 117(1), 67-86. doi:10.1037/00332909.117.1.67

Russell, B. S., Trudeau, J. J., \& Leland, A. J. (2015). Social influence on adolescent polysubstance use: The escalation to opioid use. Substance Use \& Misuse, 50(10), 1325-1331. doi:10.3109/10826084.2015.1013128

Shrout, P. E., \& Bolger, N. (2002). Mediation in experimental and nonexperimental studies: New procedures and recommendations. Psychological Methods, 7(4), 422-445. doi:10.1037/ 1082-989X.7.4.422

Simons-Morton, B., Haynie, D., Liu, D., Chaurasia, A., Li, K., \& Hingson, R. (2016). The effect of residence, school status, work status, and social influence on the prevalence of alcohol use among emerging adults. Journal of Studies on Alcohol and Drugs, 77(1), 121-132. doi:10.15288/jsad.2016.77.121

Starcevic, V. (2010). Problematic Internet use: A distinct disorder, a manifestation of an underlying psychopathology, or a troublesome behaviour? World Psychiatry, 9(2), 92-93. doi:10.1002/j.2051-5545.2010.tb00280.x

Starcevic, V. (2013). Is Internet addiction a useful concept? Australian and New Zealand Journal of Psychiatry, 47(1), 16-19. doi:10.1177/0004867412461693

Starcevic, V., \& Aboujaoude, E. (2017). Internet addiction: Reappraisal of an increasingly inadequate concept. CNS Spectrums, 22(1), 7-13. doi:10.1017/S1092852915000863

Starcevic, V., \& Billieux, J. (2017). Does the construct of Internet addiction reflect a single entity or a spectrum of disorders? Clinical Neuropsychiatry, 14, 5-10. Retrieved from http:/ www.clinicalneuropsychiatry.org/pdf/02Starcevic.pdf

Tucker, J. S., Pedersen, E. R., Miles, J. N., Ewing, B. A., Shih, R. A., \& D'Amico, E. J. (2014). Alcohol and marijuana use in middle school: Comparing solitary and social-only users. Journal of Adolescent Health, 55(6), 744-749. doi:10.1016/ j.jadohealth.2014.06.015

Wood, M. D., Read, J. P., Palfai, T. P., \& Stevenson, J. F. (2001). Social influence processes and college student drinking: The meditational role of alcohol outcome expectancies. Journal of Studies on Alcohol, 62(1), 32-43. doi:10.15288/jsa.2001.62.32

Wu, J. Y. W., Ko, H. C., Tung, Y. Y., \& Li, C. C. (2016). Internet use expectancy for tension reduction and disinhibition mediates the relationship between borderline personality disorder features and Internet addiction among college students: Oneyear follow-up. Computers in Human Behavior, 55, 851-855. doi:10.1016/j.chb.2015.09.047

Wu, J. Y. W., Ko, H. C., Wong, T. Y., Wu, L. A., \& Oei, T. P. (2016). Positive outcome expectancy mediates the relationship between peer influence and Internet gaming addiction among 
adolescents in Taiwan. Cyberpsychology, Behavior and Social Networking, 19(1), 49-55. doi:10.1089/cyber.2015.0345

Young, K. S. (1998). Internet addiction: The emergence of a new clinical disorder. Cyberpsychology, Behavior and Social Networking, 1(3), 237-244. doi:10.1089/cpb.1998.1.237

Young, K. S. (2007). Cognitive behavior therapy with Internet addicts: Treatment outcomes and implications. Cyberpsychology,
Behavior and Social Networking, 10(5), 671-679. doi:10.1089/ cpb.2007.9971

Zhao, F., Zhang, Z. H., Bi, L., Wu, X. S., Wang, W. J., Li, Y. F., \& Sun, Y. H. (2017). The association between life events and Internet addiction among Chinese vocational school students: The mediating role of depression. Computers in Human Behavior, 70, 30-38. doi:10.1016/j.chb.2016.12.057 